\title{
Pharmaceutical Applications of Gas Chromatography
}

\author{
Mohamed Jwaili \\ Department of Pharmaceutical Chemistry, Misurata University, Misurata, Libya \\ Email: Mohamed.Jwaili@gmail.com
}

How to cite this paper: Jwaili, M. (2019) Pharmaceutical Applications of Gas Chromatography. Open Journal of Applied Sciences, 9, 683-690.

https://doi.org/10.4236/ojapps.2019.99055

Received: May 15, 2019

Accepted: September 17, 2019

Published: September 20, 2019

Copyright $\odot 2019$ by author(s) and Scientific Research Publishing Inc. This work is licensed under the Creative Commons Attribution International License (CC BY 4.0).

http://creativecommons.org/licenses/by/4.0/ (c) (i) Open Access

\begin{abstract}
Gas chromatography is an analytical tool for the separation of compounds in complex mixtures based on the polarity of compounds. Separation is achieved only for compounds that are volatile or that can be made volatile on derivatization of the compound using derivatizing agents. This is one of the widely accepted tools for the separation of compounds because of its simplicity, sensitivity, and effectiveness. The principle of separation of compounds depends on the partitioning behaviour difference between mobile and stationary phase, the sample is carried by a moving gas stream through a tube packed with a finely divided solid or may be coated with a film of a liquid. Different types of columns having a various composition of stationary phase are been used for the separation of different classes of compounds mixture or sample in a suitable solvent is introduced through the injector maintained at higher temperature which is capable of volatilizing the compound into the column.
\end{abstract}

\section{Keywords}

Gas Chromatography, Flame Ionization Detector, Gas Chromatography Infra-Red Spectroscopy

\section{Introduction}

Sample gets adsorbed in the stationary phase of the column which is then separated by the carrier gas flowing through the column based on the polarity. The carrier gas will be an inert gas either helium or nitrogen of purity $99.999 \%$. Liquid samples are vaporized before injection into the carrier stream. The substances having the greater interaction with the stationary phase are retained for longer time in the column to a greater and consequently separate from those with smaller interaction. Thus, the compounds eluted out depending on the polarity from the column at different times are detected by detectors and an en- 
hanced signal is obtained. Different compounds have different RT based on the polarity [1]. The response of such Gas Chromatography detectors is proportional to the concentration of the analyte in the introduced sample. Various types of detectors used are Flame ionization detector (FID), thermal conductivity detector (TCD), Electron capture detector (EC), Nitrogen phosphorous detector (NPD) \& Mass detectors (single and triple quadruple modes) [2].

In combination with proper detectors selective to the type of analyte in sample gas chromatography finds application in the qualitative and quantitative analysis in various fields likes the environment, pharmaceuticals, petroleum industries \& food testing. Chemo metric analysis of headspace-GC impurity fingerprints allows distinguishing between genuine and counterfeit medicines and to differentiate between groups of counterfeit products based on the public health risks they pose [3]. The GC section of United States Pharmacopeia (USP) 27 Chapter outlines the basic theory and separation technique of GC. Various applications of GC in pharmaceutical fields are:

1) Residual solvent analysis;

2) Analysis of various functional groups;

3) Percentage of purity of pharmaceutical compounds;

4) For the analysis of drugs of abuse;

5) In pharmaceutical R \& D's to determine the identity of natural products which contains complex mixture of similar compounds;

6) In the metabolomics studies.

This assignment discusses the various applications of GC in pharmaceutical fields and the advantages and disadvantages.

\section{Various Applications of Gas Chromatography in Pharmaceutical Fields}

\subsection{Impurity Profiling}

Impurities in pharmaceutical compounds originate during the synthetic process from raw materials, intermediates and/or by-products. Impurity profiling is the process of acquiring and evaluating data that establishes biological safety of an individual impurity; thus, revealing its need and scope in pharmaceutical research. There is no clear definition for impurity in the pharmaceutical world. Residual solvents, by product, transformation products, degradation products, interaction products and related products are referred to as impurities. Impurity profiling includes identification, structure elucidation and quantitative determination of impurities and degradation products in bulk drug materials and pharmaceutical formulations. Impurity profiling has gained importance in modern pharmaceutical analysis due to the fact that unidentified, potentially toxic impurities are hazardous to health and in order to increase the safety of drug therapy, impurities should be identified and determined by selective methods. Identification of impurities is done by variety of Chromatographic and Spectroscopic techniques, either alone or in combination with other techniques. The advent of 
hyphenated techniques has revolutionized impurity profiling, by not only separation but structural identification of impurities as well.

Hyphenated techniques are mostly used for impurity determination. Hyphenated techniques are those techniques, where two or more analytical techniques are combined. The various GC-IR and GC-MS are two hyphenate techniques for this purpose. According to ICH guidelines, Impurities associated with APIs are classified into the following categories:

- Organic impurities (Process and Drug related);

- Inorganic impurities;

- Residual solvents;

- Organic impurities.

Organic impurities may arise during the manufacturing process and/or storage of the drug substance.

Starting materials, by-products, intermediates, degradation products, reagents, ligands and catalysts. Starting materials or intermediates are the most common impurities found in every A PI unless a proper care is taken in every step involved in throughout the multi-step synthesis. Although the end products are always washed with solvents, there are chances of remaining.

Thus impurity structure determination can be performed in real time during chromatographic separation and both isolation and characterization are performed in one single step. The use of hyphenated techniques for impurity determination is on rise due to easy availability of bench-top instrumentation and their distinct advantages like versatility, sensitivity, possibility of profiling sub structural analysis and rapid selective quantitative determination of targeted compound even in mixtures. The only limitation of hyphenated techniques is the heavy cost of instrumentation due to which their use is not common and spread worldwide like GC, HPLC, MS or NMR systems. As on today these sophisticated techniques are mainly used for the purpose of monitoring, characterization and identification of impurities but they can be used for other analytical purposes as well.

\subsection{Residual Solvent Analysis}

Residual solvent analysis in pharmaceuticals refers to the analysis of organic volatile impurities that are formed as by-product during the synthesis of drug products or during the packing and storage. According to GLP norms it is mandatory for drug manufacturers to ensure that these residues are removed, or are present only in limited concentrations in the products. Gas chromatography is widely being used for the residual solvent analysis. The detectors mainly used for the detection of these volatiles are flame ionization detector (FID) or Mass spectrometry (MS). Most popular sample introduction technique is by using static Head space sampler/dynamic head space analyser, solid-phase micro extraction, and direct injection of solution containing bulk drug substance or drug product into the gas chromatograph. 


\section{Static Head space analyser:}

In static head space sampler sample are placed in crimper vials and heated. The gas sample collected after the equilibration between gas and liquid (or solid) phase is reached. Volatile organic compounds get collected on the space above the sample in the vial. This is then injected into the GC using loop injection where it get separated based on the polarity of the compound and detected by the detector either FID or MS. This method is preferred when the pharmaceutical samples are soluble in solvents, such as water, DMSO, DMA, DMF, DMI or benzyl alcohol. Generally, static headspace analysis is the most widely used technique for residual solvent determination in pharmaceutical. Residual solvent analysis by static HS/GC can be enhanced by careful consideration of two basic concepts-partition coefficient $(\mathrm{K})$ and phase ratio $(\beta)$. Partition coefficients and phase ratios work together to determine the final concentration of volatile compounds in the headspace of sample vials. Volatile components partition from the sample phase and equilibrate in the vial headspace. Striving for the lowest values for both $\mathrm{K}$ and $\beta$ when preparing samples will result in higher concentrations of volatile analytes in the gas phase and, therefore, better sensitivity [4] (Figure 1).

\section{Functional Group Identification}

There are different ways of identification of functional groups in pharmaceutical compounds using GC coupled with IR and UV as detectors. Before the invention of FTIR instruments (which are fast), GC-IR was used for the identification of functional group. In this case sample injected into GC after separation using column gets deposited on to a salt window in the IR instrument giving IR absorption of the functional groups present in the. Like GC-MS, this is a separation technique followed by identification, using infrared spectroscopy. Another way

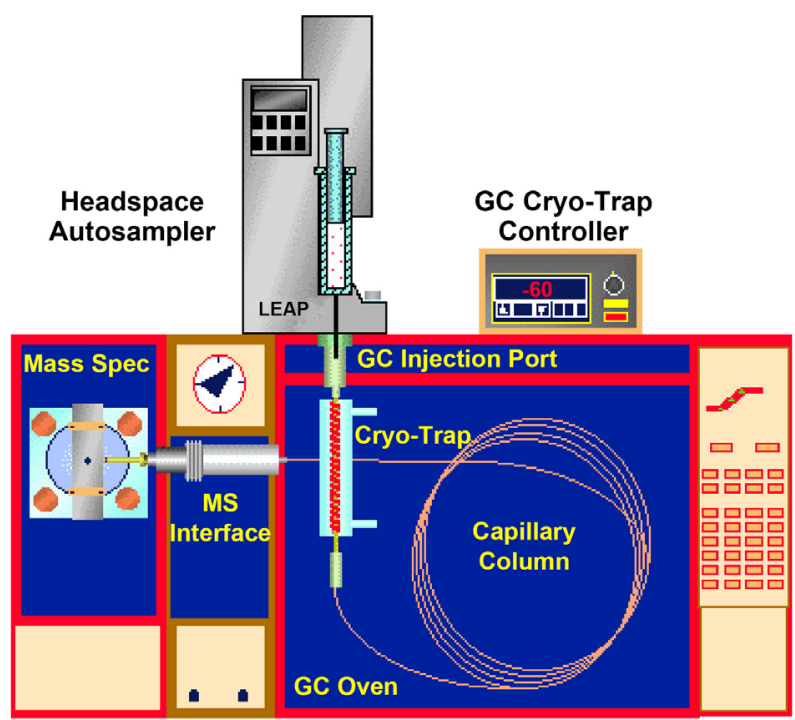

Figure 1. GC with Head space analyser. 
of identification of specific functional groups and compounds in complex mixtures is by GC-UV. Separations of compounds are carried out using a micro gas chromatograph built into a gas flow cell and using an external capillary gas chromatograph linked to the same gas flow cell. Usually the wavelength range where analysis is usually performed is $168-330 \mathrm{~nm}$ [5]. Various uses of GC-UV are the determination of cigarette smoke, petroleum, dust and flavour. Another important use of GC-UV is the identification of functional groups by comparison with reference library of spectrum.

The presence of various bioactive compounds in phytoextracts confirms the application of these compounds for various ailments. Isolation of individual phytochemical constituent may ensue to find a novel drug. Hyphenated technique called GCMS is used for identification of active principles present in the phytoextracts of long chain hydrocarbons, alcohols, acids, esters, alkaloids, steroids, amino acid and nitrocompounds [6]. Chromatogram of the compounds reveals the presence of various compounds present in the sample. From the GCMS chromatogram the active compounds with retention time RT, Molecular formula, Molecular weight and concentration percentage in various solvent extracts are determined

\section{Uses of GC in Metabolomics}

Gas chromatography coupled with mass spectrometry has been extensively used in the field of metabolomics study even though this is not been associated with study involving volatile organic compounds. Study of metabolomics is useful for Drug assessment, clinical toxicology, Nutrigenomics and functional genomics.

As a separation method GC is known for its high reproducibility and powerful resolving capability. GC is coupled to MS analysers such as the triple quadruple (QqQ), which can be used in both qualitative and quantitative analysis, and the time-of-flight (TOF) instrument which can determine accurate mass to 4 decimal places. Compounds eluting from a GC column gets ionized either by Electron ionization or chemical ionization. Application includes fields of different ranges like food industry and agriculture, to pharmaceutical and disease biomarker discovery applications [7] [8] [9] [10]. Electron ionization normally carried out at $-70 \mathrm{ev}$. This is used to ionize the samples which are then detected by mass spectrometer. Mass spectrum obtained is compared with mass spectrum in the data base and the compounds identified. Databases can be constructed which record mass spectra and also retention time to give highly accurate $2 \mathrm{D}$ databases for compounds which can be shared across analytical platforms Usually FeihnLib library is one such data base available for this purpose. The early adoption of GC-MS as a tool for metabolomics helped for the development of these types of databases). There are two types of metabolomics study targeted and none targeted. In Non-targeted metabolomics studies, which 'fingerprint' of an organism's metabolome is determined which involves, involve detection and often quantization of the maximum possible number of compounds from a sample in 
order to compare sets of samples and gain useful biological insight [11]. In targeted metabolomics profiling the compounds in a given bio fluid or tissue extract identified and quantified by comparing the spectrum of interest to a library of reference spectra of pure compounds.

Metabolomics study using GC is possible only if the compound can be volatilized or it can be derivatized to a volatile form using various types of derivatizing agents. Derivatization is the process of modifying the chemical structure of a compound to make it easy for identification by GC. There are two types of derivatizing agent's i.e. selective and non-selective derivatising reagents. These derivatizing agents help in the modification of compounds nature like volatility, improve stability and also improve chromatographic behaviour such as resolution and peak tailing. Commonly derivatives are prepared by acylation, alkylation or silylation of certain functional groups [12]. A dry step is performed prior to derivatization in the case of analysis of biological fluids as moisture can quenche the reaction. Derivatization procedure can also be done in aqueous media by selecting proper reagents. There are a lot of derivatization kits available now a days which are simple to use. Trimethylsilyl is widely used derivatization agent producing TMS derivatives. In the database of metabolomics several TMS derivatives could be found. Development new derivatisation protocols for non-volatile molecules extend the reach of this technique to a greater variety of metabolites and provide overlap with other platforms like liquid chromatography. This allows the use of instruments working in conjunction to fully map the profile of an organism or sample by analysis of metabolites with wide-ranging physiochemical properties.

Quantification of metabolites identified can also be performed either by internal or external standard method. QqQ detectors are often used for this purpose. In this method ions will get excluded selectively before and after fragmentation. This ensures that the ions reaching the detector are from the precursor ion only. This type of tandem MS experiment is referred to as selected/multiple reaction monitoring (S/MRM). This improves selectivity and sensitivity, which are highly critical for metabolite identification. If isotopically labelled internal standards is available and affordable, accurate quantisation can be performed at very low levels in complex biological samples. Other than some of the disadvantages of GC-MS such as increased sample preparation for polar molecules it has lot of advantages too i.e. it is reproducible, high sensitivity and compatibility with comprehensive libraries. These advantages of this hyphenated technique justify its continued use in metabolomics profiling.

\subsection{Analysis of Drugs of Abuse}

GCMS Coupled with Headspace makes a good analytical tool for the analysis of drugs of abuse. Analysis of Amphetamines and its metabolites in urine, Nicotine content in pharmaceutical drugs is an example for this type of analysis. GCMS coupled with chemical ionization and conventional headspace will give better 
sensitivity of about 20 times in SIM mode. GC MS produces a constant ionization making it easier for the comparison of identified compounds with the library data [13] [14] [15].

\subsection{Clinical Toxicology}

Molecular ion formation, availability of increased range of compounds that can be analysed using GC, its high sensitivity and faster analysis makes it useful in the field of clinical toxicology. Usually Supersonic GCMS finds application in clinical toxicology. This is also used for the confirmation and also rejection of LCMS analysis reports in certain cases [16] [17]. Toxins and venoms are usually identified and quantified using this method.

\section{Conclusion}

One of the major disadvantages of GC is that it is applicable only for volatile compounds or compounds that can be made volatile by chemical modification at a temperature with which it decomposes. When an unknown mixture of compounds is injected strongly retained components move slowly through the column sometimes by increasing the temperature in the temperature programming. But there is a maximum limit for column that can withstand based on the nature of the column. This is another disadvantage of Gas chromatography. Even though, GC has above said few limitations, it finds extensive application in various sectors of pharmaceutical and clinical field both in research and quality control purposes like quality assurances, production, pilot plant developments for active pharmaceutical ingredients, bulk drugs and formulations. It is also used for the identification of impurity components in the drug synthesis, pharmacognosy pharmaceutical process control and pharmaceutical biotechnology due to its high detector sensitivity and high resolving power.

\section{Conflicts of Interest}

The author declares no conflicts of interest regarding the publication of this paper.

\section{References}

[1] Jennings, W., Mittlefehldt, E. and Stremple, P. (1987) Analytical Gas Chromatography. Academic Press, San Diego.

[2] Phd, K.T. (2004) Quality-Control Analytical Methods: Gas Chromatography. International Journal of Pharmaceutical Compounding, 8, 305-309.

[3] Custers, D., Canfyn, M., Courselle, P., De Beer, J.O., Apers, S. and Deconinck, E. (2014) Headspace-Gas Chromatographic Fingerprints to Discriminate and Classify Counterfeit Medicines. Talanta, 123, 78-88.

https://doi.org/10.1016/j.talanta.2014.01.020

[4] Chauhan, A., Goyal, M.K. and Chauhan, P. (2014) GCMS Technique and Its Analytical Application in Science and Technology. Journal of Analytical and Bioanalytical Techniques, 5, 222. https://doi.org/10.4172/2155-9872.1000222 
[5] Lagessona, V., Lagesson-Andraskoa, L., Andraskob, J. and Bacoc, F. (2000) Identification of Compounds and Specific Functional Groups in the Wavelength Region 168-330 nm Using Gas Chromatography with UV Detection. Journal of Chromatography $A, 867,187-206$. https://doi.org/10.1016/S0021-9673(99)01123-1

[6] Muthulakshmi, A., Margret, J.R. and Mohan, V.R. (2012) GC-MS Analysis of Bioactive Components of Feroniaelephantum Correa (Rutaceae). Journal of Applied Pharmaceutical Science, 2, 69-74.

[7] Bartolozzi, F., et al. (1997) Simultaneous Determination of Soluble Sugars and Organic Acids as Their Trimethylsilyl Derivatives in Apricot Fruits by Gas-Liquid Chromatography. Journal of Chromatography A, 758, 99-107. https://doi.org/10.1016/S0021-9673(96)00709-1

[8] Capitan, P., et al. (1999) Gas Chromatographic-Mass Spectrometric Analysis of Stable Isotopes of Cysteine and Glutathione in Biological Samples. Journal of Chromatography B: Biomedical Sciences and Applications, 732, 127-135. https://doi.org/10.1016/S0378-4347(99)00273-X

[9] Kudo, K., et al. (2012) Rapid and Reliable Screening Method for Detection of 70 Pesticides in Whole Blood by Gas Chromatography-Mass Spectrometry Using a Constructed Calibration-Locking Database. Legal Medicine, 14, 93-100. https://doi.org/10.1016/j.legalmed.2012.01.002

[10] Tsugawa, H., et al. (2014) Highly Sensitive and Selective Analysis of Widely Targeted Metabolomics Using Gas Chromatography/Triple-Quadruple Mass Spectrometry. Journal of Bioscience and Bioengineering, 117, 122-128. https://doi.org/10.1016/j.jbiosc.2013.06.009

[11] Dunn, W.B., et al. (2011) Procedures for Large-Scale Metabolic Profiling of Serum and Plasma Using Gas Chromatography and Liquid Chromatography Coupled to Mass Spectrometry. Nature Protocols, 6, 1060-1083. https://doi.org/10.1038/nprot.2011.335

[12] Kind, T., et al. (2009) FiehnLib: Mass Spectral and Retention Index Libraries for Metabolomics Based on Quadruple and Time-of-Flight Gas Chromatography/Mass Spectrometry. Analytical Chemistry, 81, 10038-10048. https://doi.org/10.1021/ac9019522

[13] Eiceman, G.A., Hill, H.H. and Gardea, T.J. (2000) Gas Chromatography. Analytical Chemistry, 72, 137-144. https://doi.org/10.1021/a10000054

[14] US Pharmacopeia Convention, Inc. (2001) United States Pharmacopeia (USP) 27. US Pharmacopeia Convention, Inc., Rockville.

[15] Hoffman, E. and Stroobant, V. (2007) Mass Spectrometry Principles and Applications. John Wiley \& Sons, Hoboken.

[16] McMaster, M. and McMaster, C. (1998) GC/MS: A Practical Users Guide. John Wiley \& Sons, New York.

[17] Keulemans, A.I. (1959) Gas Chromatography. Reinhold Publishing Corp., New York. 